\title{
LES REGARDS PHOTOGRAPHIQUES COMME DISPOSITIFS ANTHROPOTECHNIQUES ${ }^{1}$
}

\author{
Paul Vancassel $^{2}$
}

Le renouvellement des méthodes utilisées dans l'étude des phénomènes sociaux (notamment avec Harold Garfinkel et l'ethnométhodologie ${ }^{3}$, la sociologie de l'action ou encore les théories de l'action située) autorise une nouvelle approche de la photographie en tant qu'activité. Parallèlement, le développement d'une anthropologie des techniques (Bruno Latour, Jean-Louis Déotte) permet d'envisager les regards photographiques comme autant d'actualisations de dispositifs anthropotechniques.

C'est plus généralement une étude de l'inscription culturelle de la photographie et des démarches photographiques qui est envisageable à partir d'une étude des regards. Ceux-ci ne se limitent pas aux «points

1 Ces remarques sont extraites de la thèse de doctorat de Paul Vancassel en Sciences de l'Information et de la Communication, soutenue en février 2008 à l'Université de Rennes 2 (« Les regards photographiques : dispositifs anthropotechniques et processus transindividuels »).

2 PREFics, Université Rennes 2 - France (pvancassel@ hotmail.com).

3 Avec Harold Garfinkel, qui rompt avec la tradition sociologique issue de Durkheim, «le fait social n'est pas un objet stable, il est le produit de l'activité continue des hommes, qui mettent en œuvre des savoir-faire, des procédures, des règles de conduites, bref, une méthodologie profane dont l'analyse constitue la véritable tâche de la sociologie» (A. Coulon, L'ethnométhodologie, Paris, PUF, coll. "Que sais-je ?", 1987, p. 19).

Recherches en communication, $\mathrm{n}^{\circ} 27$ (2007). 
de vue » des photographes, mais sont davantage des processus transindividuels d'organisation du réel liés à des pratiques, à des postures et à des usages signifiants d'un point de vue sociohistorique.

Cette approche doit ainsi permettre de prendre en compte les différents niveaux avec lesquels l'activité photographique dialogue dans son développement. Au-delà des images et des appareils utilisés, les significations associées aux usages de ce procédé s'inscrivent aussi dans des champs médiatiques où les regards photographiques font sens. En tant qu'expérience, chacun d'eux se développe en mobilisant des significations et en se référant à des modèles qui excèdent largement le seul domaine de la production iconique.

Une approche anthropologique dynamique de la photographie possède également des conséquences pour la théorie de l'information : celle-ci n'est plus simplement un message déjà constitué, mais renvoie à un processus d'organisation et d'institutionnalisation d'un sens. Dans une telle perspective, l'information émerge à partir des interactions entre les différents éléments constitutifs des dispositifs.

\section{Principes d'une étude des regards photographiques comme dispositifs anthropotechniques}

Nous proposons d'envisager les regards photographiques comme des activités et des expériences qui, en tant que telles, s'appuient sur des éléments collectifs et culturels. Ils ne sont pas seulement les résultats des intentions des photographes, mais, plus généralement, d'un ensemble d'éléments hétérogènes qui forment un dispositif, au sens foucaldien ${ }^{1}$. Ce dispositif comprend, par exemple, des conventions de composition, des formats techniques, des filières industrielles et commerciales, des systèmes de diffusion, des savoir-faire, des comportements et des rôles professionnels, des habitudes sociales, des valeurs accordées à l'obser-

1 Selon Michel Foucault, un dispositif « est un ensemble résolument hétérogène, comportant des discours, des institutions, des aménagements architecturaux, des décisions réglementaires, des lois, des mesures administratives, des énoncés scientifiques, des propositions philosophiques, morales, philanthropiques, bref : du dit, aussi bien que du non-dit (...). Le dispositif lui-même, c'est le réseau qu'on peut établir entre ces éléments.» (M. FOUCAULT, entretien avec Alain Grosrichard, « Le jeu de Michel Foucault », Ornicar ?, n 10, Paris, 1977, p. 63). 
vation visuelle... Ces éléments collectifs ne s'opposent pas à l'intervention du photographe, mais exigent que l'on resitue ses choix dans un cadre de communication sociale. Par ailleurs, les regards photographiques ne sont pas réductibles aux seuls éléments photographiés (aux thèmes et aux sujets des photographes) ou aux seules images produites, qui ne seraient pas, dans ce cas, des signes. Pour que les images photographiques fassent sens, il faut qu'elles soient liées à un ensemble d'éléments sociaux et non visuels, qui sont associés à leur production et lui donne leur sens.

Notre hypothèse est donc qu'au-delà de tel ou tel photographe qui actualise un regard particulier, existent aussi des regards photographiques comme activités socialement signifiantes et expériences culturellement situées, à travers lesquelles, à chaque époque depuis l'invention de la photographie, se construisent des façons de se rapporter au réel et de le mettre en images.

\section{Toute expérience est liée à une situation}

Nous partons du principe pragmatique de John Dewey que toute expérience admet une situation comme corrélat : une situation qualitative et qualificatrice est présente comme arrière-plan et contrôle de toutes les expériences. ${ }^{1}$ Pour lui, une situation est par la suite un environnement privilégié, dans lequel les acteurs sont concrètement engagés. Elle possède la qualité d'être «expériencée » par eux. ${ }^{2}$ A chaque époque, nous envisageons ainsi un ensemble d'éléments concrets qui constituent l'activité photographique comme la mise en œuvre d'un dispositif anthropotechnique. Chaque situation ne se comprend pas uniquement comme l'environnement matériel du photographe (allant des appareils utilisés au contexte de la prise de vue), mais désigne aussi un ensemble de conditions concrètes de possibilités, à la fois techniques, visuelles, historiques, médiatiques et culturelles. On peut aussi qualifier ces conditions de possibilité d' "efficaces », afin de préciser qu'elles pèsent effectivement sur le développement des regards des photographes.

1 J. DEWEY, Logique, la théorie de l'enquête, Paris, PUF, 1993, p. 131.

2 Ibid. 


\section{Une situation produit localement de l'ordre}

La théorie dite de "l'action située » nous conduit plus généralement à réévaluer la notion d' " acte photographique » (ses conditions, ses acteurs, mais aussi sa dynamique) à partir de la prise en compte de l'ensemble des éléments concrets sur lesquels repose son exercice. Contre une conception de l'action comme réalisation d'une délibération rationnelle par un acteur désincarné, cette approche pragmatique insiste sur le fait qu'une situation produit localement de l'ordre, qu'elle implique une expérience et qu'elle redéfinit concrètement les modalités de la cognition. Dans ce modèle de "l'action située », l'acteur n'est plus le siège exclusif de la capacité d'agir, du contrôle de l'activité ni de la cognition. Incarné, il partage ces attributs avec les objets, les artefacts, les outils et les non-humains en général. ${ }^{1}$ Dans cette perspective, l'analyse de l'action souligne que celle-ci ne peut pas être déterminée en dehors de l'engagement effectif des acteurs dans la situation. Le contrôle de l'action n'est plus le seul résultat d'une préparation mentale (et préalable) de l'action, mais son effectuation concrète en contexte. ${ }^{2}$ Nous proposons d'envisager ainsi les regards photographiques comme autant d'activités qui mobilisent des ensembles complexes d'éléments qui constituent des « situations photographiques ». La notion de « dispositif anthropotechnique » nous permet d'envisager cet ensemble d'éléments (dont certains échappent à la volonté du photographe) qui se coordonnent entre eux.

\section{L'expérience photographique est à la fois active et passive}

La théorie de « l'action située » nous conduit à envisager les différentes « expériences photographiques » qui constituent les regards photographiques. Dans la perspective de John Dewey, la notion d' « expérience » renvoie à un processus, à la fois actif et passif, qui se développe dans la durée. ${ }^{3}$ Ensuite, l'expérience photographique peut désigner aussi

1 L. QUÉRÉ, «La situation toujours négligée ? », Réseaux, n85, Paris, CNET, sept.oct. 1997, p. 179.

2 Idem, p. 169.

3 «L'expérience humaine est constituée, de part en part, de contextes historiques, sociaux et politiques. (...) En anglais, comme nom et comme verbe, l'expérience désigne à la fois un événement accompli et un processus ; elle enveloppe à la fois 
bien «l'expérience éprouvée » et vécue par le photographe que «l'expérimentation » qu'il construit à travers son activité photographique. Dans les deux cas, chaque regard photographique apparaît comme une confrontation avec la réalité, qui apporte son lot de problèmes et au cours de laquelle le photographe essaie des solutions techniques, mais subit aussi des contraintes. L'expérience photographique est ainsi marquée par une implication, mais aussi par le développement d'un ensemble de gestes et de choix de la part du photographe, qui sont aussi des adaptations, des façons pour lui de «se laisser envahir » par différents niveaux de sa réalité (c'est-à-dire de la situation photographique dans laquelle il est engagé). L'expérience photographique est autant une « mise en œuvre » qu'une « mise à l'épreuve »1. Avec John Dewey, l'expérience est orientée vers un équilibre, vers la composition d'un « tout intégré », par réduction des incertitudes, des tensions et des incompatibilités. Chaque regard photographique est également orienté vers la production d'un ensemble d'images, qui tendent à manifester clairement les choix de leur auteur.

\section{Repenser la dimension technique de la photographie}

Dans le cadre d'une approche anthropotechnique, la relation de l'individu et de l'appareil photographique sera reconsidérée comme un agencement. Les regards photographiques ne sont pas les regards de «sujets-photographes » à travers des appareils, mais émergent des situations dans lesquelles ils s'exercent, et dont les photographes et les appareils font partie. L'appareil n'est pas un simple instrument, mais est aussi associé à des pratiques sociales et à des schèmes cognitifs à partir desquels il est institué comme appareil. Par ailleurs, la technicité de l'appareil ne saurait se réduire au schéma optique de la camera

l'instant immédiat et la durée» (R. SHUSTERMAN, Préface à J. DEWEY, L'art comme expérience, Publications de l'Université de Pau / éditions Farrago, 2005, p. 13).

1 Pour John Dewey, «il n'y a pas d'expérience, d'expérience structurée, sans une articulation étroite d'un subir et d'un faire, sans la fusion d'une passivité («somewhat experienced ») et d'une activité ( some processes of experiencing ») : il s'agit d'une connexion, pas seulement d'une alternance. L'agent lui-même est directement affecté par ses propres actions, et c'est par ce biais qu'il peut les contrôler et les ajuster à la situation» (A. OGIEN et L. QUÉRÉ, Le vocabulaire de la sociologie de l'action, Paris, Ellips»es, 2005, p. 40). 
obscura, mais doit être pensée comme la corrélation technologique de plusieurs éléments (tels que le support d'enregistrement, l'objectif, l'obturateur, le système de visée...). Il s'insère aussi lui-même dans un système technique plus large. ${ }^{1}$

\section{La dimension transindividuelle de l'objet technique}

Compris comme une médiation entre l'individuel et le collectif, l'objet technique est aussi et surtout le modèle d'une dimension « transindividuelle ». Par la notion de «transindividualité », Gilbert Simondon précise le mode spécifique de relation interhumaine qu'introduit l'objet technique. Il s'agit d'une relation qui ne met pas les individus en rapport au moyen de leur individualité constituée les séparant les uns des autres, ni au moyen de ce qu'il y a d'identique en tout sujet humain, par exemple les formes a priori de la sensibilité, mais au moyen de cette charge de nature qui est conservée avec l'être individuel, et qui contient potentiels et virtualités. ${ }^{2}$ L'appareil photographique est indissociable de tout un ensemble de savoir-faire, d'attitudes visuelles, de toute une culture comprise comme un ensemble de potentialités, qui sont véhiculées à chaque époque par les regards photographiques. En effet, la « réalité transindividuelle » n'est ni d'origine sociale ni d'origine individuelle : elle est déposée dans l'individu, portée par lui, mais elle ne lui appartient pas et ne fait pas partie de son système d'être comme individu. ${ }^{3}$ Cette dimension transindividuelle permet d'appréhender chaque regard photographique autrement que comme une opération de mise en forme du réel par le photographe : chaque regard s'individualise à partir de potentialités dont l'individu est porteur, mais qui ne lui appartiennent pas en propre.

1 P. FLICHY, in P. BÉAUD, L. QUÉRÉ et alii., Sociologie de la communication, Paris, Réseaux / CNET, 1997, p. 252.

2 G. SimONDON, L'individuation psychique et collective, Paris, Aubier, 1989, p. 248.

3 Idem, p. 193. 


\section{Le cadre de référence sociotechnique}

L'objet technique doit plus généralement être étudié comme un objet social : il ne doit plus être étudié indépendamment de ses usages et de ses utilisateurs. Mais l'objet technique peut aussi s'articuler au social à partir de l'étude de l'imaginaire qui lui est associé. Les discours qui accompagnent le développement des nouvelles techniques jouent également un rôle en tant que médiations qui lient le technique et le social, et permettent notamment de mobiliser différents acteurs (ingénieurs, politiques, usagers innovateurs, mais aussi commerçants...) Selon Patrice Flichy, l'inscription sociale des activités techniques conduit à envisager " un cadre de référence socio-technique », qui précisera une définition commune aux différents acteurs. Ce cadre possède deux aspects : on peut s'y rapporter en tant que «cadre de fonctionnement », mais il constitue également « un cadre d'usage $»^{1}$. Chaque aspect peut être envisagé pour lui-même : d'une part, le cadre de fonctionnement valorise certains savoir-faire, délimite des usages potentiels ${ }^{2}$ et, d'autre part, les premières utilisations vont amener une modification du cadre d'usage. ${ }^{3}$ Mais le "cadre de référence socio-technique » est surtout socialement construit à travers une série de médiations. ${ }^{4}$ De façon comparable, nous envisageons les regards photographiques comme les corrélats de «stabilisations » historiquement situées, qui sont autant de « cadres de référence anthropotechniques ». Ceux-ci sont les résultats d'interactions entre des données technologiques (les différents dispositifs photographiques), des usages sociaux, des imaginaires associés et des cadres de diffusion médiatiques (qui influencent aussi les imaginaires, en tant que ces cadres de diffusion sont des cadres d'inscription ou des « arrière-plans » pour les photographes).

1 On peut parler d'un cadre de fonctionnement et d'un cadre d'usage qui sont unis par des liens analogues à ceux qui associent le signifié et le signifiant en sémiologie (Patrice Flichy, op. cit., p. 251).

2 Idem, p. 260.

3 Idem, p. 254.

4 Idem, p. 256. 


\section{L'arrière-plan sociohistorique des dispositifs anthropotech- niques}

En définitive, l'examen des regards photographiques comme dispositifs anthropotechniques nous conduit à examiner un arrièreplan sociohistorique sur lequel ils prennent appui, ainsi que la relation dynamique qui se développe entre ces deux entités. C'est à partir de cet arrière-plan sociohistorique qu'ils pourront être compris comme des phénomènes transindividuels, c'est-à-dire comme des phénomènes portés par les individus et ne leur appartenant pas en propre. En ce qui concerne les regards photographiques, l'enjeu est dès lors de savoir à quelle conception du «monde social » on aboutit lorsqu'on envisage la diversité des éléments (à la fois pratiques et théoriques) qui sont mobilisés par eux ou susceptibles de l'être. Comment ce monde sociohistorique fonctionne-t-il et permet-il la stabilisation des regards photographiques ? La prise en compte des structures spécifiques qui définissent ce monde sociohistorique a notamment été le souci de Wilhelm Dilthey, en particulier dans son dernier ouvrage inachevé L'édification du monde historique dans les sciences de l'esprit ${ }^{1}$. Il y souligne la nécessité, pour l'historien et le sociologue, de partir d'une compréhension de l'expérience concrète de ce qui constitue le « sens commun » social. Dans cet ouvrage, il envisage également la démarche compréhensive à partir de l'étude des «productions symboliques de la vie sociale». Selon lui, les « sciences de l'esprit » ont pour objet privilégié les « extériorisations de la vie » et notamment les médiations symboliques. La saisie de ces productions symboliques doit supposer un accès interne aux systèmes de significations qui les sous-tendent ${ }^{2}$, mais qui demeurent inaccessibles aux perceptions sensorielles. D'un point de vue méthodologique, il se démarque ainsi, dans ses derniers travaux, d'une " conception psychologique de la compréhension », pour envisager une orientation « ensembliste » dans la démarche des sciences de l'esprit : comprendre équivaut désormais à intégrer les éléments du donné dans une totalité où ils prennent sens. ${ }^{3}$ En tant que productions symboliques, les

1 Inachevé et publié d'abord en 1910, L'édification du monde historique dans les sciences de l'esprit a été traduit en français par Sylvie Mesure et publié par les éditions du Cerf en 1988.

2 N. ZACCAÏ-REYNERS, Le monde de la vie, tome 1, « Dilthey et Husserl », Paris, Cerf, 1995, p. 19.

3 S. MESURE, présentation de W. Dilthey, L'édification du monde historique dans les 
regards photographiques se constituent en situation et à partir d'une expérience vécue, comme les rencontres d'activités individuelles avec des éléments socioculturels immédiatement saisis et qui pèsent sur eux. Chaque dispositif anthropotechnique est ainsi en quelque sorte un « creuset » où les « éléments du donné » (facteurs sociaux, cadres de diffusion possibles, formes diverses...) trouvent leur sens en s'intégrant dans la visée unificatrice d'un regard photographique, qui peut dès lors être compris aussi comme « horizon».

\section{Exemples de regards photographiques envisagés comme dispositifs anthropotechniques}

Il ne nous sera pas possible d'envisager ici un grand nombre d'exemples de regards photographiques, empruntés à toute l'histoire de la photographie, ce qui fait l'objet de notre thèse. Nous nous contenterons d'envisager le regard développé par Albert Londe (qui collabore, à partir de 1882, avec le professeur Charcot à l'Hôpital de la Salpêtrière), ainsi que celui mis en œuvre par Walker Evans (qui collabore avec le projet du gouvernement américain connu sous le nom de Farm Security Administration, entre 1935 et 1937). Notre éclairage sur ces deux exemples n'a pas pour prétention de mettre à jour des nouvelles connaissances historiques, mais de proposer une interprétation des éléments culturels et socio-historiques qui influencent chaque regard photographique, afin d'illustrer notre propos.

\section{La photographie instantanée comme dispositif anthropo- technique}

En tant que résultat d'un dispositif anthropotechnique, le regard photographique d'Albert Londe est d'abord déterminé par le développement de la photographie instantanée, et notamment du gélatinobromure d'argent. Mis au point par l'anglais Richard Leach Maddox en 1871, ce support d'enregistrement ne sera utilisé en France de manière

sciences de l'esprit, op. cit., p. 23. 
courante qu'à partir du début des années $1880^{1}$. La photographie instantanée permet alors le développement de nouveaux champs d'investigation pour les regards et renouvelle les visibilités. Apparu d'abord sur des plaques de verre, le gélatino-bromure d'argent est plus sensible à la lumière que le collodion et permet d'abréger le temps de pose. Mais, étant aussi chimiquement plus stable que celui-ci, il permet également de préparer les plaques à l'avance. Il remet en cause l'unité du processus de production artisanal des premiers photographes : il permet la séparation dans le temps de la préparation, de l'impression et du développement de la plaque sensible. Sa diffusion s'accompagne ainsi plus fondamentalement d'une évolution des pratiques. La plupart du temps utilisées dans des appareils moyens formats, les plaques au gélatinobromure d'argent permettent un renouvellement des conditions du geste photographique. Ce sont aussi les mécanismes des appareils qui évoluent, entraînant également une modification des gestes associés à la prise de vue elle-même : l'obturation et l'ouverture ne se font plus à la main (comme c'était le cas avec les dispositifs photographiques précédents, où l'opérateur enlevait et replaçait un bouchon devant l'objectif), mais au moyen d'un obturateur. Dès lors, la photographie instantanée implique que les durées d'exposition soient déterminées par des tables de calculs. On voit apparaître un grand nombre de manuels, à l'intention des amateurs, où sont précisés les temps d'exposition conseillés en fonction des sujets photographiés. Le développement d'un nouveau dispositif technologique est donc indissociable d'un ensemble de gestes, de méthodes, de comportements et de savoir-faire, qui possèdent une dimension sociale. Mais la photographie instantanée bouleverse également les habitudes visuelles des contemporains. Par la diminution des temps d'exposition qu'elle autorise, elle dispense également de la pose. Une autre conséquence fondamentale de l'introduction de la photographie instantanée est l'apparition d'images d'individus « pris sur le vif », auxquelles les contemporains n'étaient absolument pas préparés. L'inscription sociale de la photographie instantanée renvoie ainsi à un dispositif au sens foucaldien, qui comprend un ensemble hétérogènes d'éléments (composé aussi bien de méthodes, de représentations, de discours, que de non-dits et de visibilités).

1 A GUNTHERT, « Esthétique de l'occasion », Études photographiques, n 9 , Paris, SFP, mai 2001, p. 85 ; A. GUNTHERT, La conquête de l'instantané, thèse de doctorat, EHESS, 1999. 


\section{Une pratique expérimentale}

L'essentiel de l'arrière-plan sociohistorique des regards qu'autorise la photographie instantanée à la fin du XIXe siècle est à comprendre dans une référence à la « méthode expérimentale » de Claude Bernard, dont L'introduction à la médecine expérimentale est publiée en 1865. Non seulement la photographie instantanée permet de voir le réel d'une manière scientifique ${ }^{1}$, mais la mise en œuvre du processus photographique s'apparente alors à la construction d'une expérience scientifique, contrôlée de part en part par l'expérimentateur. Par ailleurs, du point de vue du résultat, le fait que l'individu arrive à extrapoler l'ensemble d'un mouvement à partir d'une seule position du sujet, suppose qu'il envisage d'abord l'idée d'un découpage expérimental de l'action en instants ponctuels. C'est une approche expérimentale de l'action qui permet d'envisager l'instant photographié comme partie d'un continuum temporel dont il est extrait, plutôt que comme résultat d'un processus déformant ou mensonger. Et si le regard photographique peut être compris comme un élargissement de la vision binoculaire, c'est parce qu'est déjà admise l'idée d'une vraie réalité de la vie mouvante ${ }^{2}$, distincte de la réalité perceptible à l'œil nu. La photographie instantanée ne se contente pas d'introduire pour l'homme un nouveau rapport au temps, mais lui permet surtout de développer son rapport visuel au monde selon une démarche expérimentale quasi-scientifique. Du point de vue transindividuel, les regards photographiques se chargent de cette signification expérimentale et scientifique, comme en témoignent quantité d'images d'amateurs, à partir de 1890, où les individus sont saisis « au vol », en train de sauter, et qui sont manifestement des illustrations d' « expérimentations photographiques » construites en vue de ce résultat. Le fait de pouvoir photographier l'individu « en l'air » est alors envisagé comme un «fait scientifique », que les amateurs reproduisent, de la même manière que l'on peut répéter une expérimentation scientifique, dans certaines conditions, à l'infini.

1 J-C. GAUTRAND, « Photographier à l'improviste, impressions instantanées », in M. FRIZOT (dir.), Nouvelle histoire de la photographie, Paris, Bordas, 1994, p. 233.

2 M. FRIZOT, «Instantané et mouvement », Histoire de voir, Paris, CNP/ Photopoche, 1989. 


\section{Un contexte médiatique}

La valeur scientifique de la photographie instantanée est ellemême liée à une diffusion médiatique. Dès 1876-1878, l'américain Eadweard Muybridge avait réalisé des photographies, grâce au collodion, qui présentaient une décomposition du trot du cheval. En 1878, à Palo Alto, il construit un véritable dispositif expérimental utilisant le $1 / 25$ e de seconde, constitué de douze appareils en batterie à obturateur à fente et dont le déclenchement était commandé par la rupture de fils tendus au travers de la piste au passage du cheval. Diffusés dans la revue La Nature du 14 décembre 1878, ces photographies ont été très commentées à l'époque. Et, plus que les images, ce sont les conditions expérimentales de leur réalisation qui ont retenu l'attention. On sait en particulier que c'est après avoir vu ces images que le physiologiste Jules Marey s'orientera également dès 1881 vers un enregistrement du mouvement par la photographie instantanée.

\section{La valeur de l'observation dans les sciences}

L'introduction de la photographie instantanée s'inscrit aussi plus généralement, pour les scientifiques, dans un débat sur les sources de la connaissance. Alors que la physiologie du XIXe siècle privilégiait l'écoute du corps, notamment à travers l'auscultation, certains scientifiques, tels Jules Marey, ont cherché à substituer à l'oreille, parfois défectueuse, la méthode de la transcription optique. ${ }^{1} \mathrm{La}$ " méthode graphique », qu'il développe dans les années 1880, porte alors la promesse d'une véritable "langue universelle », car sans intermédiaires : elle ne concerne pas d'abord la photographie, mais l'usage de nombreux appareils enregistreurs, produisant des tracés et permettant aux scientifiques d'obtenir des notations détachées de l'observateur. Les enregistrements chronophotographiques dérivent de cette méthode graphique, et doivent permettre aux choses et aux corps de " s'autoécrire eux-mêmes ». Les regards photographiques qui se développent autour de 1880 s'appuient ainsi sur un arrière-plan socioculturel essentiellement lié à la culture scientifique d'alors, bien plus qu'à une « tradition photographique ».

1 D. BERNARD et A. GUNTHERT, L'instant rêvé d'Albert Londe, Nîmes, Jacqueline Chambon, 1993 


\section{La photographie médicale d'Albert Londe}

Albert Londe s'inscrit dans ce contexte d'une utilisation scientifique de la photographie. Il s'agit pour lui, au moyen de ce procédé, d'observer, sonder, mesurer (...) les corps : en extraire de nouvelles visibilités, de nouveaux savoirs. ${ }^{1}$ Par là, il agit bien dans une démarche inspirée de la "méthode expérimentale » de Claude Bernard, dans la mesure où il s'agit de mettre en évidence des faits. Il convient cependant de souligner qu'il se heurte alors à la conception de l'observation qui avait été développée auparavant par le professeur Charcot. Lorsqu'il le rejoint en 1882, celui-ci était en effet reconnu comme le promoteur d'un recours à l'observation dans le travail scientifique (notamment dans l'étude de l'hystérie), mais qui était essentiellement une « observation sur le vif ». Cette conception de l'observation était au fondement de sa clinique et permettait des « leçons de choses » dans ses enseignements. Et cette conception s'opposait alors, dans son principe, à celle développée par la physiologie expérimentale, qui construisait au contraire des « observations provoquées $» .^{2}$ L'arrivée d'Albert Londe à la Salpêtrière est liée à un changement de méthode chez Charcot. ${ }^{3}$ Mais il semble que le photographe ait été beaucoup plus enthousiaste que lui sur la valeur « scientifique » de ces photographies. Bien qu'il présente parfois des images réalisées par Albert Londe (notamment lors d'une leçon sur l'hystérie masculine en 1882), Charcot n'a pratiquement pas utilisé ces photographies dans ses textes et ses publications. Cela nous permet d'affirmer que le regard photographique d'Albert Londe se heurte, en tant qu'activité, à une autre conception, déjà constituée, de l'observation scientifique : le regard du clinicien « sur le vif». Si son regard photographique s'appuie sur la reconnaissance de la «méthode

1 A. ROUILLÉ, La photographie, Paris, Gallimard, 2005, p. 96.

2 Alors que Bernard vise à subordonner la pathologie à la physiologie, l'observation à l'expérimentation, Charcot ne cessera de défendre la clinique contre le laboratoire, le médecin contre l'homme de système (D. BERNARD et A. GUNTHERT, op. cit., p. 104).

$3 \mathrm{Au}$ cours de 1878, Charcot met au point un protocole à la fois clinique et expérimental, en recourant à l'hypnose, afin de provoquer artificiellement des états nerveux qui se développent spontanément chez l'hystérique. À partir de 1882, Albert Londe entreprend d'enregistrer ces manifestations de l'hystérie, afin de documenter les recherches de Charcot. Il fabrique même, à partir de 1883, un appareil chronophotographique à neuf, puis à douze objectifs, pour enregistrer les différents instants d'une crise hystérique. 
expérimentale », il est donc aussi une activité dont la portée heuristique est limitée dans le domaine scientifique. Cette méfiance des scientifiques à l'égard des photographies peut d'ailleurs être liée à un privilège des sources écrites dans le travail scientifique ${ }^{1}$, mais aussi, plus fondamentalement, à une définition traditionnelle du savoir scientifique comme « connaissance de l'universel » depuis Aristote. Cependant, la réticence des scientifiques est aussi une condition socioculturelle du regard d'Albert Londe, puisqu'elle le pousse à développer un programme pour une application de la photographie au domaine médical. ${ }^{2}$

\section{Le regard photographique de Walker Evans}

Notre deuxième exemple propose d'envisager le regard photographique de Walker Evans et de mettre à jour le dispositif anthropotechnique sur lequel il repose. Ce photographe américain commence son activité en 1929, après un séjour en France (1926-1927), où il étudie l'œuvre de Gustave Flaubert à la Sorbonne. En tant que photographe, il fut d'abord influencé par la Nouvelle Vision européenne, construisant des compositions géométriques (privilégiant des angles élaborés) et illustrant les thèmes de la modernité (grues, chantiers, gratte-ciel, câblages métalliques du pont de Brooklyn). Mais son regard évolue radicalement à partir de 1930, lorsqu'il fait l'acquisition d'une chambre photographique : il s'oriente alors vers des vues plus descriptives et plus frontales, caractéristiques de ce dispositif technologique, qui impose de travailler «sur pied ». Cette orientation technique correspond aussi pour lui à une volonté de s'opposer à une photographie dite « artistique » et de lui préférer une photographie strictement documentaire, débarrassée de tout « sentimentalisme ». ${ }^{3}$ Dès 1931, il appelle, dans la revue Hound \& Horn, à un «processus clinique » de description de la société américaine par la photographie. Se détournant des images de gratte-ciel, il réalise, toujours en 1931, une série de vues de façades de maisons victoriennes des environs de Boston. À l'occasion de ce reportage de commande, il développe les bases d'une véritable « forme

1 J.-P. TERRENOIRE, dans «Images et sciences sociales : l'objet et l'outil », Revue française de sociologie, XXVI, 1985, pp. 509-527.

2 A. LONDE, La photographie médicale, Paris, Gonthier-Villars, 1892.

3 O. LUGON, Le style documentaire, d'Auguste Sander à Walker Evans, Paris, Macula, 2000 ; S. MARESCA, La photographie, miroir des sciences sociales, Paris, L'Harmattan, 1997, p. 94. 
documentaire », reposant sur des cadrages simples et une forte luminosité, à même de souligner les détails. En 1934, dans une lettre, il énonce à nouveau son projet d'une description exhaustive de la société américaine dans ses aspects les plus ordinaires. À l'instar d'Auguste Sander, il est attentif aux «types » qui se manifestent à travers les êtres particuliers ; mais il s'intéresse aussi à des aspects de la vie sociale qui ne sont pas présentés par la presse (ce projet aboutira en 1938 à l'exposition American Photographs, présentée au MOMA de New-York). Ainsi, avant d'entrer à la F.S.A. (avec laquelle sa collaboration est d'ailleurs fréquemment remise en cause), Walker Evans a déjà défini son projet personnel. Il utilisera sans doute les moyens que lui offre cette administration, sans adhérer totalement au projet de l'agence, qu'il assimile à de la propagande.

\section{L'absence d'implication de l'auteur}

Mais Walker Evans trouve également la justification de son regard dans des éléments non-photographiques. Il gardera ainsi toute sa vie une admiration pour Gustave Flaubert : l'esthétique de Flaubert est absolument la mienne (...) son réalisme et son naturalisme, et son objectivité de traitement : la non-apparition de l'auteur, la non-subjectivité. ${ }^{1}$ Cette esthétique lui permet de développer « l'absence d'implication » (disinterested $^{2}$ ) du photographe, une quasi-disparition de l'auteur : Flaubert affirme qu'il ne faut pas s'écrire. ${ }^{3}$ Elle lui permet de fonder un regard qui ne favorise pas l'affectivité. Le personnage d'Emma Bovary est l'exemple type de ce qu'il faut éviter : sa personnalité trouble sa vision du monde. Elle voit mal car elle cherche le bonheur dans l'émotion, dans l'exaltation de la sensibilité et des sens. ${ }^{4}$ À l'inverse, l'artiste est ouvert au spectacle du monde quel qu'il soit car, solitaire, il sait s'en retirer d'une certaine façon même lorsqu'il y est présent. ${ }^{5}$ Par ailleurs, l'intérêt qu'il développe pour une esthétique de la simple description

1 Walker Evans, cité par O. LUGON, op. cit., p. 78.

2 Ce terme a été utilisé dans l'introduction du livre American Photographs, publié en 1961 ; cité par O. LUGON, op. cit., p. 33.

3 Gustave Flaubert, Lettre à Mlle Leroyer de Chantepie, 18 mars 1857 ; citée par G. SÉGINGER, Flaubert, une éthique de l'art pur, Lièges, Sedes, 2000, p. 71.

4 G. SÉGINGER, op. cit., p. 70.

5 Ibid. 
est aussi à mettre en relation avec l'intérêt que manifestent des universitaires américains dans les années 1930 (les sociologues notamment) pour l'art et la culture vernaculaire : architecture provinciale, enseignes publicitaires, décorations d'intérieur, par exemple, sont soudain mis en valeur aux États-Unis. Toutes ces productions semblent appréhendées comme autant de manifestations d'un " art anonyme » qui est pour Evans, non seulement un thème, mais un modèle. ${ }^{1} \mathrm{Il}$ parle d' " arrangements inconscients » pour désigner ces compositions qui s'organiseraient en quelque sorte d'elles-mêmes dans la vie sociale. La recherche d'une photographie impersonnelle va de pair avec l'idée que l'environnement humain est lui-même un arrangement qui résulte de la vie quotidienne. S'il s'oppose à une « photographie d'art » (ou plutôt s'il se désintéresse de toute option formelle en photographie), c'est donc au nom d'une autre conception de l'art, qu'il juge en quelque sorte plus légitime ou plus authentique : la culture littéraire ou la culture vernaculaire.

\section{Le regard documentaire comme dispositif fictionnel}

Enfin, le projet photographique de Walker Evans peut être interprété comme l'affirmation d'une « option archivale ». Le reportage documentaire qu'il pratique s'écarte de la seule représentation de l'actualité et s'adresse à un « spectateur à venir ». ${ }^{2} \mathrm{Il}$ nous inviterait ainsi à contempler une époque à l'instant qui précède son effondrement. ${ }^{3}$ On retrouve cette " orientation photographique » à la même époque chez Berenice Abbott. Ce regard n'implique pas un engagement du photographe pour la sauvegarde des objets montrés, qu'il faudrait « sauver de l'oubli » pour les générations à venir. Bien plus, il semble que la figure du «spectateur de l'avenir » soit davantage une instance imaginaire opérant dans le présent. ${ }^{4} \mathrm{Ce}$ regard documentaire, caractérisé par

1 O. LUGON, op. cit., p. 148.

2 Selon Olivier Lugon, "le spectateur supposé des images documentaires serait moins le contemporain qu'un public à venir auquel elles seraient chargées de fournir des informations historiques sur l'époque photographiée" (O. LUGON, op. cit., p. 333).

3 L. Kirstein, postface à Walker Evans, 1938 ; cité par O. LUGON, op. cit., p. 344.

4 Selon Olivier Lugon, "la figure du spectateur de l'avenir pour lequel on prétend travailler paraît constituer, plus que le destinataire des images, une instance imaginaire opérant dans le présent, un modèle de regard pour la contemplation immédiate. C'est au contemporain que, par ce renvoi constant, on demande d'endosser le regard de 
un « intérêt archéologique » pour le présent, est la construction d'une fiction. En tant que dispositif anthropotechnique, il présuppose également l'arrière-plan de la presse illustrée, dont il entend se démarquer. Si Walker Evans critique une "photographie d'actualité », celle-ci est aussi un élément de la situation photographique où son regard apparaît. ${ }^{1}$ Le regard photographique de Walker Evans s'explique donc à partir d'un ensemble d'éléments hétérogènes qui forment un autre dispositif anthropotechnique que celui qui était à l'origine du regard d'Albert Londe.

\section{Conclusion}

Les regards photographiques ne sont pas seulement déterminés par des facteurs technologiques (les différents appareils utilisés). Ils renvoient en réalité à des dispositifs anthropotechniques, qui comprennent des savoir-faire, des représentations socioculturelles ou des cadres de diffusion. Chacun d'eux se développe, en tant qu'activité, dans une situation photographique particulière, qui dépasse l'environnement immédiat du photographe et est aussi un cadre anthropologique spécifique.

Médiations entre l'individuel et le collectif, ils sont les affirmations concrètes de « visibilités », qui trouvent leur sens dans une culture en grande partie non-photographique (qui peut être aussi bien scientifique que littéraire) et à laquelle chaque photographe se réfère. Mais les regards photographiques sont plus que des productions symboliques de chaque époque de l'Histoire : en tant qu'actualisations de dispositifs anthropotechniques, ils demandent à être expliqués à partir d'arrièreplans plus vastes que les seuls moments de leurs mises en œuvre.

En tant que "projets situés », les regards photographiques ne se réduisent pas à des ensembles d'images. Leurs valeurs et leurs significations impliquent de prendre en compte les systèmes de significations

la postérité : lui-même devrait porter au présent un intérêt d'ordre archéologique, éprouver une sorte de nostalgie instantanée" (O. LUGON, op. cit., p. 343).

1 "C'est en partie parce qu'il a pu faire l'apprentissage de sa propre position de spectateur de l'avenir que le contemporain est en mesure de projeter sur son époque un regard surplombant" (Ibid, p. 357). 
qui sous-tendent les pratiques photographiques et sur lesquels elles prennent appui.

Et, en tant qu'ils sont « expériencés », ils désignent des formes de vie, qui dépendent aussi bien des biographies des photographes que de la société à laquelle ils participent. Dans leur fonctionnement, ce sont des processus transindividuels, par lesquels les individus sont porteurs de significations dont ils ne sont pas l'origine, mais plutôt les catalyseurs.

Les regards photographiques indiquent ainsi, en dernier lieu, des processus à partir desquels les individus précisent à chaque époque les modalités de leur attestation de la réalité signifiante. Ils permettent ainsi, à travers l'étude des dispositifs anthroptechniques auxquels ils sont associés, d'envisager les interactions concrètes à l'œuvre dans la production de l'information et l'organisation du sens. 